\title{
Cancer metabolic and immune reprogramming: the intimate interaction between cancer cells and microenvironment
}

\begin{abstract}
Cancers are highly complex tissues characterized by dynamic and reciprocal interactions between tumor cells and their microenvironment. During tumor progression, pervasive stromal reprogramming and remodeling transform a normal to a tumorigenic microenvironment. Heterotypic cooperation between tumor cells and immune/ inflammatory and stromal cells allows selection of more aggressive cancer cells that develop important adaptive strategies. Among these strategies, metabolic and immune reprogramming has been recently highlighted. Overcoming metabolic stress is a critical step for tumor growth. Tumor cells shift towards a high rate of glycolysis and induce aerobic glycolysis in neighboring fibroblasts cells in type of host-parasite relationship. Recent data showed that sub-populations of tumor cells overexpress innate and/or adaptive immune markers like Toll-like receptors, immunoglobulin molecules and recombination activating genes 1 and 2, which are normally restricted to immune system cells. Accumulating evidence suggest that this expression can promote tumor growth and survival. This review focuses on the current knowledge in glucose metabolic and immune reprogramming during cancer progression.
\end{abstract}

Keywords: cancer cells, tumour microenvironment, cancer adaptive strategies, metabolic reprogramming, immune reprogramming
Volume I Issue 2 - 2014

Didier Meseure, Kinan Drak Alsibai

Platform of Investigative Pathology and Department of Tumor Biopathology, Curie Institute, France

Correspondence: Didier Meseure, Platform of Investigative Pathology and Department of Tumor Biopathology, Curie Institute, F-75248, Paris Cedex 05, France, Tel 33-0-172-38-9397, Email Didier.meseure@curie.fr

Received: June 09, 2014 | Published: August 02, 2014
Abbreviations: ECM, extracellular matrix; CAFs, cancer associated fibroblasts; TLRs, toll-like receptors; Ig, immunoglobulin; RAG, recombination activating genes; TCA, tri carboxylic acid; MCT, monocarboxylate transporter; ROS, reactive oxygen species; HK2, hexokinase 2; PET, positron emission tomography; PFK-1, 6-phosphofructo-1-kinase; PK, pyruvate kinase; SPs, specificity proteins; GFs, growth factors; PDGF, platelet-derived growth factor; TGF- $\beta$, transforming growth factor- $\beta$; bFGF, basic fibroblast growth factor; EMT, epithelial-to-mesenchymal transition; TGF $\beta 1$, transforming growth factor- $\beta 1$; MDSCs, myeloid-derived suppressor cells

\section{Introduction}

Two of the most fundamental requirements to normal tissue survival are the ability to turn over nutrition to generate energy and develop an effective response against pathogenic agents. In contrast, cancers are highly complex tissues in which tumor cells interact with stromal cells within a modified extracellular matrix (ECM). ${ }^{1}$ This tumorigenic microenvironment is composed of a variety of mesenchymal immune/inflammatory and non-immune stromal cells Heterotypic interactions between stromal cells and tumor cells with genetic and epigenetic alterations generate acquisition of numerous adaptive strategies, resulting in diversification of tumor cell phenotypes and tumor heterogeneity. ${ }^{2}$ Among these adaptive strategies, we can distinguish plasticity, acquisition of stem-like properties, unfolded protein response, autophagy, and production of exosomes, invasion, metabolic reprogramming, immunosuppression and therapeutic resistance. ${ }^{3}$ The importance of metabolic and immune reprogramming in cancer progression has been highlighted recently. ${ }^{4}$ This review focuses on the current knowledge in cancer glucose metabolic and immune reprogramming and discusses how tumor cells directly and indirectly interact with the tumorigenic microenvironment.

\section{Cancer metabolic reprogramming}

Metabolic alterations observed in cancer are adaptation mechanisms to support the anabolic requirements of tumor cells, or they arise from oncogenic alterations in metabolic enzymes themselves. ${ }^{5}$ In order to survive and grow in host tissue, tumor cells consume glucose at high rates even in the presence of adequate oxygen, an effect first described by Warburg in the last century. Hence, aerobic glycolysis became a cancer hallmark. In fact, cancer cells are surrounded by stromal cells within ECM, supported by a vascular network. These stromal cells are forced to produce energy-rich nutrients to feed tumor cells. Thus, stromal cells could influence the metabolism of adjacent epithelial tumor cells by a mechanism of stromal-epithelial metabolic coupling. ${ }^{6}$ In this scenario, hypoxia and reactive oxygen species ${ }^{\mathrm{ROS}}$ exert a key role in affecting cancer metabolism.

\section{Glucose transport in cancer cells}

Glucose is transported into the cell by GLUTs, which are present in all types of cells, and have a variable affinity for glucose. The GLUT families comprise 14 known members of proteins (GLUT1 to GLUT14) that mediate glucose transport through the cell membrane. Functionally, the GLUTs regulate the movement of glucose between the extracellular medium and the intracellular compartments, maintaining the glucose supply available for cell metabolism. ${ }^{7}$ GLUT1, 2, 3 and 12 were the main glucose transporters in human tumor. ${ }^{8,9}$ GLUT1, 5 and 12 expressions were detected in invasive and noninvasive breast carcinomas, while it was absent in adjacent normal breast tissue ${ }^{10-12}$ GLUT3 is induced during epithelial-mesenchymal transition (EMT) and promotes tumor cell proliferation in non-small cell lung cancer. ${ }^{13}$

\section{Glycolysis in cancer cells}

Normal cells obtain only $10 \%$ of their energy by Glycolysis, with the remainder being the result of mitochondrial respiratory activity. 
Under normoxic conditions the glycolytic pathway involves the metabolism of glucose to 2 molecules of pyruvate, and $2 \mathrm{NADH}, \mathrm{H}+$ with a net gain of 2 ATP. Then, pyruvate enters the mitochondria and decarboxylated by pyruvate dehydrogenase into acetyl-CoA allowing it to enter the tricarboxylic (TCA) cycle. ${ }^{14}$ Under the influence of hypoxia, glucose is catabolized to lactate via glycolysis to yield ATP; mitochondria cannot produce ATP in oxidative phosphorylation without oxygen to aid in electron transport. This type of production is termed "Pasteur effect". The monocarboxylate transporter MCT4 mediates lactic acid efflux from most tissues that are dependent on glycolysis for their ATP production. MTC4 is up-regulated by hypoxia through a HIF-1 $\alpha$-mediated mechanism. This adaptive response allows the increased lactic acid produced during hypoxia to be rapidly lost from the cell. ${ }^{15}$

In contrast, tumor cells have a high rate of glucose uptake and glycolytic metabolism. They obtain the majority of their energy by glycolysis, maintaining elevated rates of lactate production, which is sufficient for tumor cell survival in a hypoxic environment. Normal mitochondrial respiration cannot occur without oxygen, thus tumor hypoxia will cause a shift to glycolysis. Since glycolysis generates a net gain of only 2 ATP per glucose molecule, a markedly smaller amount of energy compared with the net gain of 38 molecules of ATP produced by respiration. Thus, to obtain sufficient energy, tumor cells require an increased glucose uptake.

The reactions catalyzed by hexokinase, phosphofructokinase, and pyruvate kinase are the major sites of regulation of glycolysis in tumor tissue.

Hexokinase: There are 4 mammalian hexokinases 1-4. The hexokinase 2 (HK2), which is bound to the outer mitochondrial membrane, is frequently over expressed in tumor cells. HK2 is often the major isoform overexpressed and is required to maintain high levels of glycolysis. In practice, the hyperactivity of HK2 in cancers is the basis of positron emission tomography (PET) that is used for primary and metastatic cancer detection. ${ }^{16,17}$ There is a significant association between the expression of GLUT1 and HK2 in cholangiocellular carcinoma, indicating that GLUT1 is major glucose transporter expressed in this tumor and that HK2 contributes to the increased metabolism of glucose. ${ }^{9}$

Phosphofructokinase: 6-phosphofructo-1-kinase (PFK-1) is the major rate-limiting step of glycolysis. Three isoforms of mammalian PFK-1 encoded by different genes have been identified; P (in brain), $\mathrm{M}$ (in muscle), and L (in liver). ${ }^{17}$ PFK-1 activity is controlled by the intracellular ATP/AMP ratio, and high levels of ATP inhibit PFK-1 activity. Fructose 2, 6-bisphosphate (F2,6BP) is a powerful allosteric regulator that acts by increasing the affinity of PFK-1 for fructose 6-phosphate and decreasing the inhibitory effect of ATP. ${ }^{18}$ The bifunctional enzyme 6-phosphofructo-2-kinase/fructose2,6-bisphosphatase (PFK-2/FBPase) determines the steady-state concentration of F2,6BP. PFK-2/FBPase is expressed in several tissue-specific isoforms encoded by at least 4 genes PFKB1-4 that are all stimulated by hypoxia through hypoxia-response elements in their promoters. Several data revealed overexpression of PFKFB-4 mRNA and protein in breast and colon cancer cells and in melanoma. ${ }^{19,20}$

Pyruvate Kinase: Pyruvate kinase (PK) is the final rate-limiting step of glycolysis. There are 4 PK isoforms in mammals (L, R, M1, and M2) encoded by 2 genes, PKL encoding the L and R isoforms and PKM encoding the M1 and M2 isoforms. In contrast to normal cells, tumor cells express exclusively PKM2. Indeed, it has recently been shown that switching PKM2 expression to PKM1 in tumor cells reverses the Warburg effect and reduces the ability of the cells to form tumors in nude mice, indicating that PKM2 expression is necessary for aerobic glycolysis and maximal tumor growth. Moreover, the tyrosine kinase signaling regulates PKM2 activity. ${ }^{21}$

\section{Warburg effect}

Tumor cells have a different metabolic profile from that of normal cells. They shift towards a high rate of glycolysis. Otto Warburg described tumor cells in mouse as having increased glycolysis and lactate production, compared to normal hepatocytes and renal cells in the presence of oxygen. ${ }^{22}$ Tumor cells frequently use glutamine as another source of fuel. Glutamine enters the mitochondria and can be used to replenish Krebs cycle intermediates. It can be used to produce more pyruvate through the action of malic enzyme. Highly proliferative cells need to produce excess lipid, nucleotide, and amino acids for the creation of new biomass. Excess glucose is diverted through the pentose phosphate shunt (PPS) to create nucleotides. Fatty acids are critical for new membrane production and are synthesized from citrate in the cytosol through the action of ATPcitrate lyase (ACL) to generate acetyl-CoA. This process requires NADPH reducing equivalents, which can be generated through the actions of malic enzyme, IDH1, and also from multiple steps within the PPS pathway. Serine and glycine are critical for biosynthesis of nucleic acids and lipids as well as proteins. ${ }^{5,23}$

Several signaling pathways are implicated in Warburg Effect. Growth factor stimulation results in signaling through receptor tyrosine kinases (RTKs) to activate PI3K/Akt and Ras. Akt promotes glucose transporter activity and stimulates glycolysis through activation of several glycolytic enzymes including HK and PFK. Akt phosphorylation of apoptotic proteins such as Bax makes cancer cells resistant to apoptosis and helps stabilize the outer mitochondrial membrane by promoting attachment of mitochondrial HK to the voltage-dependent anion channel complex, the major channel of the mitochondrial outer membrane. RTK signaling to c-Myc results in transcriptional activation of numerous genes involved in glycolysis and lactate production. The p53 oncogene transactivates TP-53induced glycolysis and apoptosis regulator (TIGAR) and results in increased NADPH production by PPS. ${ }^{24}$

\section{Reverse Warburg effect}

Fibroblasts represent the principal cellular component of tumor microenvironment. Once they are recruited, activated, and accumulated to the tumor microenvironment, these cells become cancer associated fibroblasts (CAFs) or myofibroblasts, and have distinctly different morphological and functional features from their normal counterparts. ${ }^{25} \mathrm{CAFs}$ are a heterogeneous cell group with various sources. The locally resident stromal fibroblasts are considered a major source of CAFs. This process is called fibroblast to myofibroblast transdifferentiation (FMT). It is activated by growth factors such as transforming growth factor- $\beta$ (TGF- $\beta$ ), platelet-derived growth factor (PDGF), and basic fibroblast growth factor (bFGF) released by the tumor cells. ${ }^{26,29}$ Bone marrow-derived mesenchymal stem cells (MSCs) serve as another important origin for CAFs, contributing approximately $25 \%$ to the CAF population. ${ }^{30}$ Several studies suggest that CAFs are derived from malignant epithelial cells via EMT. ${ }^{31}$ and endothelial cells via endothelial to mesenchymal transition (EndoMT). A transgenic mouse model showed that up to $40 \%$ of CAFs arose from endothelial cells at the invasive front of tumors. ${ }^{32} \mathrm{CAFs}$ also originate from other cell types including smooth muscle cells, pericytes, adipocytes, or inflammatory cells. ${ }^{33}$ 
Growing evidence highlights the role of CAFs supporting the metabolic reprogramming of tumor cells. Recently, it has been proposed that tumor epithelial cells also induce aerobic glycolysis in neighboring fibroblasts. ${ }^{34}$ Then, CAFs undergo FMT and secrete lactate and pyruvate that could be taken up by tumor cells. ${ }^{35}$ In this model, tumor cells use CAFs for providing them high energetic sources and transform the normal stroma into a wound healing-like stroma. When tumor cells are co-cultured with fibroblasts tumor cells increase their mitochondrial mass, while fibroblasts lose their mitochondria. Oxidative stress in fibroblasts induces the autophagic destruction of mitochondria by mitophagy. Then, stromal cells are forced to undergo aerobic glycolysis, and produce energyrich nutrients (such as lactate and ketones) to feed tumor cells. To accomplish this metabolic coupling, CAFs export lactate and ketone bodies via MCT4 transporters. In turn, tumor cells import and recycle these fuels via the MCT1 transporter to produce ATP. MartinezOustschoorn et al., ${ }^{36}$ suggest a new scenario called "reverse Warburg effect" in which the stromal catabolism fuels the anabolic growth of tumor cells, via autophagy and mitophagy, promoting tumor progression and metastasis. ${ }^{34}$ They described another phenomenon in which cancer cells metabolically reprogram fibroblasts resulting in "metabolic asymmetry" between cancer cells and fibroblasts. In this asymmetric tumor metabolism, cancer cells are anabolic due to catabolic tumor fibroblasts. ${ }^{37}$

The lactate uptake by cancer cells increases the surrounding $\mathrm{pH}$, thus also protecting cells from the extremely acid microenvironment. Lactate can also stimulate the expression of hyaluronan and hyaluronidase in CAFs and tumor cells which can increase cancer invasion and metastasis. ${ }^{38}$ Consistently, oxidative stress in CAFs leads to the up-regulation of MCT4, which shuttles lactate to the epithelial cells. $^{39}$

\section{ROS and HIF-I involvement in cancer metabolism}

In tumor cells, ROS accumulate abnormally and induce signaling cascades that mediate the oncogenic phenotype. In addition to their impact on tumor cells, ROS also affect the surrounding cells that constitute the tumor microenvironment. ROS production increases tumor angiogenesis, drives the onset of inflammation and promotes FMT. Indeed, ROS have been implicated in the metabolic reprogramming of both cancer cells and CAFs, allowing an adaptation to oxidative stress that ultimately promotes tumorigenesis and chemoresistance. ${ }^{35}$ It is also recognized that metabolic reprogramming is under control of oxidative stress and hypoxia. The transcription factors HIF-1 and HIF-2 play also a crucial role in the metabolic reprogramming of both CAFs and tumor cells. Although their role remains controversial under hypoxia, clear evidence now exist indicating that ROS stabilize the HIF proteins under normoxic conditions through modulation of PHD enzyme activity ${ }^{40-44}$ Fiaschi and Chiarugi ${ }^{43}$ have shown that accumulation of ROS and HIF stabilization in CAFs result also from the down-regulation of SIRT3, a mitochondrial NAD-dependent deacetylase. HIF through SIRT3 can regulate CAFs metabolism driving the Warburg effect. In addition, HIF-1 regulates several genes involved in glucose metabolism, as GLUT1 and GLUT3 in order to increase the glucose uptake in the cell. Moreover, the HIF-1a promoter contains binding sites for several transcriptional factors, including SP1 (61). HIF targets MCT4, responsible for the export of lactate and activates PKM2 transcription, contributing to reprogram the glucose metabolism of epithelial cells (8, 62). Moreover, OCT1 which is a transcription factor, may cooperate with p53 in regulating the balance between oxidative and glycolytic metabolism. OCT1 regulates a set of genes that increase glucose metabolism and reduce mitochondrial respiration. One of these genes encodes pyruvate dehydrogenase kinase, isozyme 4 (PDK4) that are activated by HIF-1. ${ }^{24}$

In summary, oxidative stress induced by ROS production has severe implications in CAFs metabolism. It drives aerobic glycolysis that in turn has profound effects on tumor microenvironment. CAFs feed tumor cells with high-rich compounds, and induce anti-oxidant defense in tumor cells allowing them to proliferate..$^{35}$

\section{The role of specificity proteins}

Numerous mammalian genes are regulated by specificity proteins (SPs), often in cooperation with other transcription factors. It has been shown that SP1 and SP3 bind to the mouse GLUT3 gene, SP1 mediating suppression and SP3 mediating activation of expression of this gene. ${ }^{44-46} \mathrm{~A}$ variety of cancers have been shown to overexpress SP, particularly SP1 and to a lesser extent SP $3 .{ }^{47}$ SPs are known to play a role in the regulation of multiple oncogenes and tumor suppressor genes as well as a number of cell cycle, apoptosis, and angiogenesis genes. ${ }^{44,47}$ There is evidence for a role of SP1 and SP3 transcription factors, in conjunction with the constitutive activation of the PI3K/ Akt signaling pathway, in regulating the abnormal glycolytic and lipogenic activity of cancer cells. ${ }^{17}$

\section{Metabolic reprogramming via PI3K/Akt/mTORCI activation}

Altered metabolism in tumor cells is not a passive reaction to damaged mitochondria, but rather an active oncogene-induced metabolic reprogrammation that allows cancer cells to support proliferation, survival and differentiation. ${ }^{4}$ Oncogenes and oncosuppressors alterations (activation of PI3K/Akt/mTORC1, Myc and Kras, loss of 53) reprogram cancer cells metabolism by increasing glucose and glutamine uptake, promoting mitochondrial biogenesis and regulating transcription and translation. Activation of the $\mathrm{PI} 3 \mathrm{~K} /$ Akt pathway is perhaps the most common lesion in human cancers. ${ }^{5}$ This activation leads to enhanced glucose uptake and glycolysis. ${ }^{48}$ The PI3K/Akt pathway also promotes glucose carbon flux into biosynthetic pathways that rely upon functional mitochondrial metabolism. Several findings demonstrate that the reprogramming of mitochondrial citrate metabolism is a central aspect of PI3K/Akt oncogenic activity.

Interestingly, oncogene activation in tumor cells is sufficient to induce the metabolic reprogramming of CAFs toward glycolysis, via oxidative stress. It appears that oncogenes also act at a distance, via ROS production and inflammation, to induce metabolic symbiosis between tumor cells and the surrounding stroma. As a consequence, some oncogenes (Ras and NF-kB) act via oxidative stress to upregulate MCT4 in the tumor stroma. ${ }^{36}$

mTORC1 (mammalian target of rapamycin complex 1) is a protein complex that functions as energy/redox sensor and controls protein synthesis. mTORC1 has been shown to have direct effects on promoting mitochondrial biogenesis, in part via a transcriptional complex that promotes the function of peroxisome proliferator-activated receptor gamma co-activator (PGC-1 $\alpha){ }^{49,50}$ It has been established a causal link between aberrant mTOR activation and tumorigenesis, indicating that mTOR inhibition may have therapeutic potential. ${ }^{51}$

\section{Cancer immune reprogramming}

Immune system is composed of innate immunity that is an early, general response against infecting agents, and adaptive immunity that provides specific protection against pathogenic agents. Innate 
immune responses are triggered by variety of pathogens, and involve the activation of polymorphonuclear leukocytes (PMLs), monocytes, macrophages, dendritic cells (DCs), natural killer (NK) cells and complements. Innate immunity to microbial pathogens relies on the specific host-receptor detection of pathogen-associated molecular patterns (PAMPs) and damage-associated molecular patterns (DAMPs). DCs and macrophages, express a number of germlineencoded pattern recognition receptors (PRRs) that specifically recognize and ingest pattern recognition molecules (PAMPs and DAMPs) and release cytokines that attract secondary, active and defensive cells from the blood.

There are at least three mechanisms in which immune responses protect normal tissue from tumor formation: (i) both adaptive and innate immunity, which can suppress viral infections that have the potential for direct cellular transformation; (ii), immunosurveillance, which involves cooperation between both types of immunity, identifies and eliminates transformed cells before they can establish malignancy; (iii) pathogen removal by the immune system reduces opportunities for inflammation, which is also considered a source of tumor development. ${ }^{52}$

In contrast, once tumors are developed, the inflammatory response changes. As a result, a set of immune cells that are immunomodulatory, rather than inflammatory, can enhance tumor progression and metastasis. They do so by promoting angiogenesis, enhancing cell migration, invasion and intravasation and suppressing antitumor immunity. ${ }^{52}$ The interaction between tumor cells and immune cells within tumor microenvironment can be distinguished in three stages; recruitment, education and response. Tumor cells recruit immune cells to the tumor microenvironment through the production of chemokines. Then, tumor cells polarize immune cells toward tumorsupporting cells through the secretion of cytokines that regulate immune cells differentiation, as well as acquire immune tolerance. Theses differentiated immune cells promote tumor growth and immune tolerance through the production of growth hormones, growth factors, chemokines and cytokines in the tumor microenvironment. ${ }^{53}$

Surprisingly enough, sub-populations of tumor cells overexpress innate and/or adaptive immune markers like Toll-like receptors (TLR), immunoglobulin molecules (Ig) and recombination activating genes 1 and 2 (RAG1 and RAG2), which are normally restricted to the immune system cells.

\section{Interplay between TLRs and epithelial-stromal- immune coupling}

The TLRs, which provide front-line protection against pathogens through recognition PAMPs, assure rapid inflammatory responses as well as communications with adaptive immunity cells. Similarly, TLRs play an active role in carcinogenesis and tumor progression during chronic inflammation that involves the tumor microenvironment DAMPs derived from injured normal epithelial cells and necrotic cancer cells appear to be present at significant levels in the tumor microenvironment, and their stimulation of specific TLRs can foster chronic inflammation. ${ }^{54}$ TLRs are expressed on many types of cancer cells, tumor stromal cells and infiltrating immune cells. TLRs that are expressed on immune and tumor cells are activated by PAMPs and DAMPs. These activated cells release cytokines and chemokines that are important components of the tumor microenvironment. Furthermore, cytokine-activated infiltrating immune cells can subsequently induce further cytokines and impair the function of antigen-presenting cells (APCs) and tumor-associated antigen (TAA)specific immunity, thereby resulting in tumor immunotolerance.
The subsequent activation of TLRs in cancer cells and the ensuing signaling cascade with the production of cytokines and chemokines may promote cancer cell survival and chemoresistance and, therefore, tumor progression. ${ }^{53}$ Recently, a direct regulatory relationship between the inducible p53 and the innate immune pathway has been established..$^{55}$ The expression of most TLR genes in human primary and cancer cells was directly affected by changes in $\mathrm{p} 53 .{ }^{52,56}$

\section{Immunoglobulin expression by tumor cells}

Traditionally, it has been believed that the production of immunoglobulin (Ig) molecules is restricted to lymphoid lineage cells. However, immunoglobulin genes and proteins have been recently found in a variety of normal epithelial cells, as well as some epithelial and non-epithelial tumor cells.

IgG heavy chain and light chain, and the immunoglobulin gene SNC73 were found to be expressed in some normal epithelial cells. ${ }^{57-59}$ Kimoto $^{60}$ have demonstrated by RT-PCR, the expression of Ig gene transcripts of $\operatorname{IgM}, \operatorname{IgD}$, IgG3, IgG1, IgE, and $\operatorname{IgA}$ in tumor cell lines of colon, breast, laryngeal, and pancreatic carcinoma. Another Ig molecules composed of $\alpha$ heavy chain and $\kappa$ light chain were also detected in several epithelial tumor cell lines, and recently in soft tissue "non-epithelial" tumor cells. ${ }^{61,62}$ The Ig expressed in tumor cells included basically all kinds of isotypes. Among heavy chains, $\alpha$ chain for IgA and $\gamma$ chain for IgG were the mostly identified. In light chain, on the other hand, only $\kappa$ chain but not $\lambda$ chain was confirmed. ${ }^{63}$

Interestingly, tumor cells also secrete assembled Ig protein out of cell membrane. By cultivating of several cancer cell lines, ELISA test reported the existence of Ig $\alpha$ heavy chain in the culture supernatant. Other supportive evidence suggested IgG secretion, the presence of IgG protein supernatant, found in the culture of uterine cervical cancer cell lines. ${ }^{64}$ Recently, transcripts of IgG1 heavy-chain constant region, immunoglobulin- $\kappa$ and $\lambda$-light chains, IgG variable region, and RAG1/RAG2 in both esophageal cancer tissues and cell lines were detected. However, activation-induced cytidine deaminase (AID) was not detected ${ }^{65}$ All these results provide the hints that in addition to Ig protein production, tumor cells are also capable of Ig secretion.

The literature about the function of Ig expressed in tumor cells is limited. Some new findings support the important role of tumor derived Ig as a growth factor of cancer cells. It could be presumed that their function might be associated with some antibody related immunological courses and result in tumor evasion from immune surveillance. Ig in tumor cells is also associated with increasing tumor grades. Tumor cell-originated IgA protein plays a role in cellular transformation. Moreover, anti-human IgA could partly inhibit cellular transformation, promote the malignant proliferation ability of cancer cells, and increase the access percentage of S phase from the early mitosis of synchronized cancer cells. Recent data demonstrated that blockade of cancer-derived Ig $\alpha$ suppressed the growth and viability of cancer cells. ${ }^{66,67}$

Similar results have been found for tumor-derived IgG. Tumor cells secreted-IgG can promote growth and survival of these cells. Blockade of tumor-derived IgG by either antisense DNA or antihuman $\mathrm{IgG}$ antibody increased programmed cell death and inhibited growth of cancer cells in vitro. The administration of antihuman IgG antibody also suppressed the growth of an IgG-secreting cancer line in immunodeficient nude mice. These results suggest also that tumor IgG expression is required, at least, in part, for survival of tumor cells. ${ }^{64}$ It has been confirmed recently that downregulation of $\operatorname{IgG}$ restrained the growth and proliferation of cancer cells in vitro and in 
vivo. It seems that $\operatorname{IgG}$ can enhance the growth and proliferation of tumor cells via inducing the production of ROS at low level. These findings provide new clues for understanding tumor proliferation and designing cancer therapy. ${ }^{68}$

Molecular mechanisms of immunoglobulin expression by tumor cells RAG1/RAG2 expression by tumor cells: RAG1 and RAG2 are indispensable for $\mathrm{V}(\mathrm{D}) \mathrm{J}$ recombination. Interestingly, both RAG1 and RAG2 mRNA and proteins have been detected in Ig positive cancer cell lines, including lung, colon, uterus cervical, liver, prostate, gastric, breast, and nasopharyngeal carcinomas and recently in thyroid and oesophageal carcinoma tissues. ${ }^{63-65,69,70}$

Activation induced cytidine deaminase (AID), which is essential for both class switch recombination and somatic hypermutation, was also reported to be expressed in six breast cancer cell lines by nested RT-PCR. ${ }^{71}$

In addition to the RAG1 and RAG2 promoters, the RAG gene has also other regulatory elements, such as the proximal enhancer (Ep), the distal enhancer (Ed) and the RAG enhancer (Erag). It is thought that the aforementioned transcription factors regulate RAG expression by binding to their corresponding regulatory sequences in $\mathrm{B}$ cells. Erag is the strongest enhancer regulating RAG expression. Targeted deletion of Erag in the mouse germline resulted in a 5-fold to 10 -fold decrease in RAG expression and a partial block at the pro-B to pre-B transition. ${ }^{72}$ E2A, Ikaros, Foxo1, Foxp1 and NF-kB were all shown to activate RAG expression by binding to Erag in murine $\mathrm{B}$ cells. Pax $5 \beta$ was reported to activate RAG2 promoter in immature $\mathrm{B}$ cells ${ }^{73}$. Chen et al. ${ }^{74}$ analyzed the protein and mRNA expressions of those transcription factors that have been found to be essential for RAG activation in B cells, including E2A (E47 and E12), Foxo1, Foxp1, Ikaros, NF- $\kappa B$, and Pax $5 \beta$, in four cancer cell lines. They studied then the localization of a number of these transcription factors (E2A, Foxp1, NF- $\kappa \mathrm{B}$ and Foxo1) by immunofluorescence. They found that E2A, Foxo1 and Foxp1 were expressed in cancer cells and localized to the nuclei of these cells. Over-expression of these three transcription factors significantly increased RAG expression. Functional inactivation of the genes of any of these three transcription factors by RNA interference decreased RAG expression. In vivo chromatin immunoprecipitation assay showed that the histone $\mathrm{H} 3$ of Erag was acetylated and that E2A, Foxo1, Foxp1 were bound to Erag in these cancer cells. These results indicate that transcription factors E2A, Foxo1 and Foxp1 activate the expression of RAG, which is critical for $\mathrm{V}(\mathrm{D}) \mathrm{J}$ recombination, in cancer.

The transcription factor B-cell lymphoma $11 \mathrm{~A}(\mathrm{Bcl11A})$ is required for $\mathrm{B}$ cell development. It is a critical component of a transcriptional network that regulates $B$ cell fate by controlling $\mathrm{V}(\mathrm{D}) \mathrm{J}$ recombination. The major B cell isoform, Bcl11A-XL, binds the RAG1 promoter and Erag enhancer to activate RAG1 and RAG2 transcription in pre-B cells. They employed Bcl11A overexpression with recombination substrates in a cultured pre-B cell line as well as Cre recombinasemediated Bcl11alox/lox deletion in explanted murine pre-B cells

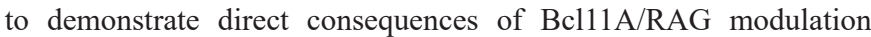
on $\mathrm{V}(\mathrm{D}) \mathrm{J}$ recombination. ${ }^{75}$ Recent data showed overexpression of Bcl11A in breast and lung cancer. ${ }^{76}$

CD40/CD40L pathway: CD40 belongs to the superfamily of tumor necrose factor (TNF) receptors. The ligand for this molecule on T-lymphocytes called CD40L. CD40 and CD40L play a key role in intercellular interactions of $\mathrm{B}$ and $\mathrm{T}$ cells of immune system. CD40 is expressed also by a number of antigen-presenting cells (DCs, monocytes, macrophages) eosinophils, basophils and also by epithelial cells. This expression possesses pronounced costimulatory properties. CD40 plays an important role in different forms of immunologic response, because it is taking part in activation, proliferation, differentiation of different types of immune cells. In normal B cells, an important mechanism of activation and Ig production is CD40 ligation-triggered cellular signaling pathways. As a consequence of CD40 signaling, a number of well-characterized signal transduction pathways are activated, including the NF- $\mathrm{kB}, \mathrm{p} 38$ mitogen-activated protein kinase, c-Jun-NH2-kinase, Janus kinases/ signal transducers and activators of transcription, and phosphoinositide 3-kinase pathways. These pathways, in turn, regulate alterations in gene expression, and induce proliferation and immunoglobulin class switching of resting B cells.

CD40 and CD40L may be expressed by a number of cancer cells. Analogously, in cancer cells, the initiation and expression of Ig gene could be regulated by the same cellular signaling pathways. Some data revealed a favorable influence of CD40/CD40L interactions on the functions of DCs, the disturbance of which happens often upon the influence of different suppressing substances produced by cancer cells. ${ }^{77}$ In addition, it has been demonstrated in breast cancer that CD40-ligation protects circulating DCs from apoptosis, by induction of expression of anti-apoptotic molecule Bcl-2. At these conditions, the functions of populations of immature DCs are promoted. It is of significance also that the activation of DCs is accompanied by the elevation of IL-1 and INF $\gamma$ production and enhanced cytotoxicity of NK cells.

Intracellular mechanism of CD40 activation is accompanied by the activation of a number of transcriptional factors of protein origin, and this process occurs with the involvement of TLR. ${ }^{78}$ However, whilst the role of CD40/CD40L interaction on immune cells has been studied in detail, the role of such interactions on tumor cells has not been clarified yet. For example, it has been demonstrated that CD40/CD40L interaction on many tumor cells (melanoma, different carcinoma, multiple myeloma, and lymphoma) may lead to inhibition of tumor cell growth, but in some cases may promote it too. ${ }^{79-82}$ The study of CD40/CD40L interaction upon cancer development is a comparatively new discipline that is actively developed, it possesses important aspects and promotes new approaches to immunotherapy.

Virus-encoded oncoproteins: Epstein-Barr virus (EBV) infected nasopharyngeal carcinoma (NPC) cell lines model may shed a light on the mechanism of cancer Ig expression regulation. Several investigations revealed that in encoded latent membrane protein 1 of EBV (LMP1)-expressing NPC cells, Igא gene enhancers iEא and

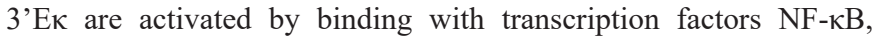
AP-1 and Ets-1, all of which were modulated by LMP1-triggered pathways. LMP1 stimulated transcription factors NF- $\kappa B$, AP-1, Ets1 binding to the corresponding site in Igא gene via NF- $\mathrm{BB}, \mathrm{JNK} /$ MAPK and ERK/MAPK signal pathways and finally upregulated Igא light chain induction. ${ }^{83}$ This presented novel proofs on the mechanisms upregulating the ectopic expression of Igא by LMP1 in NPC. Since other virus-encoded oncoproteins, such as HBX, E6, E7, can also activate many signal pathways including NF- $\mathrm{kB}, \mathrm{AP}-1$ and ERK pathways, these oncoproteins might induce Ig gene expression through the mechanism similar to EBV-LMP1.

TRL9-My88 pathway: It is now known that TLR9 agonists stimulate and increase the secretion of IgM in tumor cells, and that cancerderived IgM recognized a series of microbial antigens and some autoantigen. This secretion of cancer-derived IgM is mediated through the TLR9-MyD88 pathway. These findings indicate that tumor cells can spontaneously produce IgM with natural antibody activity. ${ }^{84}$ 


\section{Immunometabolic interactions and cancer adaptive strategies}

Several recent evidences have showed an interesting example of a link between immune system and metabolism. Recently, Tannahill et al., ${ }^{85}$ demonstrated that the metabolite succinate, a key component in the Krebs cycle, is integral in lipopolysaccharide (LPS)-induced macrophage activation, and they proved that the activation of TLR4, through LPS stimulation, leads to an increase of intracellular glutamate uptake and upregulation of succinate production under the gamma-amminobutyric acid shunt metabolic pathway. The metabolite succinate then stabilizes HIF-1 $\alpha$; a protein that is involved in interleukin-1 $\beta$ production. In addition, it has been shown that augmenting glycolytic flux drives CD8 $+\mathrm{T}$ cells toward a terminally differentiated state, while its inhibition preserves the formation of long-lived memory CD8+ T cells ${ }^{86} \mathrm{CD} 8+\mathrm{T}$ cells are recognized as cytotoxic T lymphocytes (CTLs) once they become activated and are generally classified as having a pre-defined cytotoxic role within the immune system. The immune microenvironment of tumor consists of multiple cell types, cytokines and stromal components that can further attract immune cells and guide their fate. Transforming growth factor- $\beta 1$ (TGF $\beta 1$ ), following its release from the ECM by interstitial flow-induced matrix tension and myofibroblast differentiation promotes tumor-associated regulatory $\mathrm{T}$ (Treg) cells and myeloidderived suppressor cells (MDSCs), which suppress cytotoxic $\mathrm{T}$ lymphocyte function to facilitate tumor immune escape.$^{87}$ Progressive immunosuppression is rottenly observed at advanced tumor stages, which is partially mediated by tumor-infiltrating immunosuppressive immune cells such as T reg cells, Th17 cells, regulatory DCs, macrophages, neutrophils and MDSCs. Macrophages have both proand anti-tumor actions depending on the activation signals, and have accordingly been classified into antitumor M1 and pro-tumor M2 macrophages. ${ }^{88}$ Inflammatory immune cells, when activated, display much the same metabolic profile as a glycolytic tumor cell. This involves a shift in metabolism away from oxidative phosphorylation towards aerobic glycolysis or Warburg effect. CAFs and DCs that have been activated via TLR agonist and express inducible nitric oxide synthase (iNOS) are also found to use the Warburg effect. The M1 macrophages also use the Warburg pathways to make ATP. Glycolysis and oxidative phosphorylation are linked when the pyruvate from the glycolytic pathway is used to make acetylCoA using the Krebs cycle. Immune cells that use this pathway are activated T cells, immunosuppressive M2 macrophages, and the proinflammatory Th17 T cells. Metabolism in the immune system is more than just connected to the cell's activation. Metabolism is involved in the homeostasis between immune cells as well as between immune cells and their stromal host cells. ${ }^{89}$ Dysregulation of metabolites has been found in many pathologies and the role of metabolite fluctuation caused by host immune cells versus pathogens is an active area of study.

In this regard, the concept of "immunoediting" has been proposed, which also encompasses three phases: elimination, equilibrium, and escape. Immunoediting allows tumor cells to evade immune destruction by becoming less immunogenic or more immunosuppressive. Such adaptive strategy, achieved through cell reprogramming, reflects an important property of tumors called immune-induced plasticity. This tumor immunoediting results in cellular reprogramming that may be accompanied by tumor alterations, including morphological changes, and the subsequent acquisition of a resistant phenotype which represents one of the fundamental barriers to the success of tumor immunotherapy. ${ }^{90}$ New findings have revealed that activated CD8 $+\mathrm{T}$ cells can stimulate breast cancer cells to undergo EMT and acquire the increased tumorigenic capability and chemotherapeutic resistance of breast cancer stem cells. ${ }^{91}$ These results represent the first direct demonstration that EMT may be a potential mechanism of immunoediting in cancer.Several studies have highlighted that cytoskeleton remodeling is a key determinant in the interaction between tumor cells and immune cells through immune synapse (IS) formation..$^{92}$ IS is a supramolecular structure involves $\mathrm{T}$ cell receptor, peptide-MHC complexes, modulatory receptor pairs, and adhesion molecules. In fact, EMT is associated with impairment of target susceptibility to CTL. Migratory CTLs recognize an epithelial cancer cell within a cluster of cancer cells bound through E-cadherinmediated adherens junctions and desmosomes. This recognition leads to the formation of IS between CTLs and target cancer cells. The shift in cytoskeletal organization can be used by tumor cells as an adaptive strategy to promote their resistance after CTLs selection pressure. In contrast, mesenchymal-like cancer cells are not able to organize E-cadherin-mediated stable adherens junctions and exhibit a much more labile actin cytoskeleton. ${ }^{93}$

It is now well recognized that metabolic transformation of CAFs with increased catabolism occurs via inflammation and high levels of ROS acting as the second messenger. A recent study by Valencia et al., ${ }^{94}$ established loss of p62 as a marker of metabolic asymmetry and metabolically transformed CAFs in prostate cancer. They described that CAFs are reprogrammed via loss of p62 to have reduced c-Myc and mTOR expression, leading to high levels of ROS and catabolism. Consequently, the increase in ROS drives inflammation via transforming TGF-b activation and the release of IL-6. This loss of p62 in CAFs is associated with high p62 expression in cancer cells, which proves the metabolic asymmetry phenomenon.

Moreover, the expression of indoleamine 2,3-dioxygenase (IDO), a tryptophan catabolizing enzyme, in tumors may lead to dysfunctional $\mathrm{T}$ cell response through depletion of tryptophan from the tumor microenvironment. ${ }^{95}$ IDO is also produced by MDSCs, regulatory DCs and macrophages. Silencing of IDO within the tumor using Salmonella typhimurium as a tumor-homing vector to deliver a shorthairpin RNA targeting IDO, allowed tumor infiltration of activated ROS-producing neutrophils and consequent tumor cell death. ${ }^{96}$ Investigations of IDO effector pathways suggest a role for mTOR control. ${ }^{97}$ Recent data determined that IDO activation blocked mTOR and the TCR regulatory kinase PKC-theta and stimulated autophagy. ${ }^{98}$ These studies validate IDO as a cancer target and deepen insights into how this nodal inflammatory modifier licenses progressive cancer.

\section{Immunosuppressive effects of CAFs}

CAFs may directly affect antitumor immune response by modulating the function of inflammatory cells, and/or indirectly impact immune response through recruitment of inflammatory cells (e.g. monocytes and macrophage). ${ }^{99}$

In two subsequent studies, it has been demonstrated that CAFs derived from metastatic melanomas show strong immunosuppressive activity by interfering with NK cells functions including cytotoxicity and cytokine production. ${ }^{100}$ Then, more recent study showed that a subset of CAFs expresses fibroblast activation protein- $\alpha$ (FAP). The depletion of FAP+ cells causes rapid hypoxic death of cancer and stromal cells by an interferon- $\gamma$ and TNF- $\alpha$-dependent mechanism. ${ }^{101}$ These observations advocate that CAFs play an important role in suppressing immune response.

Moreover, Tenascin C, one of the immuno-modulating ECM molecules released by CAFs, can inhibit the migration of monocytes into tumor cell areas and adhesion of $\mathrm{T}$ lymphocytes to fibronectin 
depending on integrin $\alpha 5 \beta 1$ and/or $\alpha 4 \beta 1$. $^{102,103}$ TSP-1 is another matricellular glycoprotein with immuno-modulating activity. It can affect antigen-presenting cells (monocytes, macrophages, and DCs) and $\mathrm{T}$ lymphocytes, involved in tumor-mediated immunosuppression by interaction with immune cells or activation of growth factors (e.g. TGF- $\beta$ ) to deposit in the ECM. ${ }^{104}$

CAFs secrete various growth factors and cytokines, such as TGF- $\beta$, hepatocyte growth factor (HGF), vascular endothelial growth factor (VEGF), PDGF, stromal cell-derived factor-1 (SDF$1)$, cyclooxygenase-2 (COX-2), epidermal growth factor (EGF), MCP-1, and interleukins (IL-1, IL-6 and IL-8) in an autocrine and/ or paracrine manner. These elements directly promote cancer cells growth and angiogenesis or indirectly promote de novo angiogenesis and metastatic spread by recruiting infiltration of immune cells. ${ }^{105,106}$ Several important signaling pathways are implicated in this function (e.g. TGF- $\beta /$ Smad, HGF/Met, and PDGF/PDGFR $)^{107}$ (Figure 1).

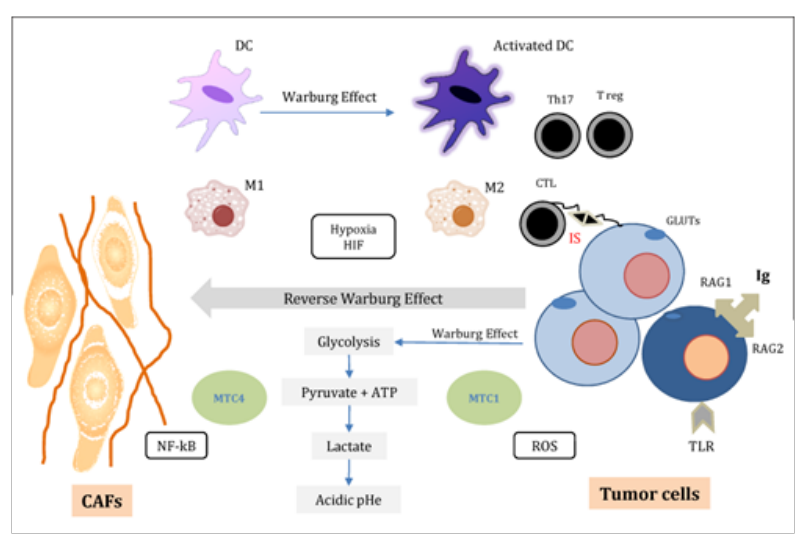

Figure I Cancer immunometabolic reprogramming.

Tumor cells undergo a specific glucose metabolic reprogramming by maintaining elevated rates of ATP, and lactate production (Warburg effect). Glucose is transported into tumor cells by GLUTs. Cancer associated fibroblasts (CAFs) feed tumor cells in type of host-parasite relationship (ReverseWarburg effect). CAFs export lactate and ketone bodies via monocarboxylate transporter MCT4. In turn, tumor cells import and recycle these fuels via the MCTI to produce ATP. This metabolic reprogramming is under control of oxidative stress, hypoxia and ROS. The transcription factors HIFI and NF-kB play a crucial role in the metabolic reprogramming of both CAFs and tumor cells. Inflammatory/ immune cells; macrophages "MI and M2", dendritic cells "DC", lymphocytes "ThI7, Treg" when activated, display much the same metabolic profile as a glycolytic tumor cell (Warburg effect). Cytotoxic T lymphocytes (CTL) recognize an epithelial tumor cell. This recognition leads to the formation of immune synapse (IS) between CTL and target tumor cells. Moreover, subpopulation of tumor cells expresses Ig, RAGI/RAG2 and/ or TLRs, which are normally restricted to immune system cells.

\section{Conclusion}

Therapy resistance is due to highly efficient adaptive strategies utilized by cancer cells. Cancer cells undergo a specific metabolic reprogramming towards opportunistic behavior, leading them to gain several advantages. Fibroblasts directly feed tumor cells in type of host-parasite relationship. Warburg effect as a well-accepted theory in cancer cells metabolism is steadily becoming established as an important phenomenon even in inflammatory/ immune responses. Activated inflammatory/ immune cells display the same metabolic profile as glycolytic tumor cells. The ability of metabolic reprogramming to modulate immune functions and the concept of immune-induced plasticity in cancer are new important clues to understand the mechanisms implicated in reciprocal cooperation between tumor cells and their microenvironment. Elucidating the tight interactions between cancer metabolic and immune reprogramming and cancer cells adaptive strategies could lead to identify pertinent tumor biomarkers and to develop new targeted therapies.

\section{Acknowledgments}

None.

\section{Conflicts of interest}

Authors declare there are no conflicts of interest.

\section{References}

1. Mueller MM, Fusenig NE. Friends or foes-bipolar effects of the tumour stroma in cancer. Nat Rev Cancer. 2004;4(11):839-849.

2. Marusyk A, Almendro V, Polyak K. Intra-tumour heterogeneity: a looking glass for cancer? Nat Rev Cancer. 2012;12(5):323-334.

3. Taddei ML, Giannoni E, Comito G, et al. Microenvironment and tumor cell plasticity: an easy way out. Cancer Lett. 2013;341(1):80-96.

4. Hanahan D, Weinberg RA. Hallmarks of cancer: the next generation. Cell. 2011;144(5):646-674.

5. Buzzai M, Bauer DE, Jones RG, et al. The glucose dependence of Akttransformed cells can be reversed by pharmacologic activation of fatty acid beta-oxidation. Oncogene. 2005;24(26):4165-4173.

6. Martinez-Outschoorn UE, Pavlides S, Howell A, et al. Stromalepithelial metabolic coupling in cancer: integrating autophagy and metabolism in the tumor microenvironment. Int J Biochem Cell Biol Jul. 2011;43(7):1045-1051.

7. Pereira KM, Chaves FN, Viana TS, et al. Oxygen metabolism in oral cancer: HIF and GLUTs (Review). Oncol Lett. 2013;6(2):311-316.

8. Ganapathy V, Thangaraju M, Prasad PD. Nutrient transporters in cancer: relevance to Warburg hypothesis and beyond. Pharmacol Ther 2009;121(1):29-40.

9. Paudyal B, Oriuchi N, Paudyal P, et al. Expression of glucose transporters and hexokinase II in cholangiocellular carcinoma compared using [18F]-2-fluro-2-deoxy-D-glucose positron emission tomography. Cancer Sci. 2008;99(2):260-266.

10. Rogers S, Docherty SE, Slavin JL, et al. Differential expression of GLUT12 in breast cancer and normal breast tissue. Cancer Lett. 2003;193(2):225-233.

11. Zamora-Leon SP, Golde DW, Concha II, et al. Expression of the fructose transporter GLUT5 in human breast cancer. Proc Natl Acad Sci U S A. 1996;93(5):1847-1852.

12. Younes M, Brown RW, Mody DR, et al. GLUT1 expression in human breast carcinoma: correlation with known prognostic markers. Anticancer Res. 1995;15(6B):2895-2898.

13. Masin M, Vazquez J, Rossi S, et al. GLUT3 is induced during epithelialmesenchymal transition and promotes tumor cell proliferation in nonsmall cell lung. Cancer Metab. 2014;2:11.

14. Palsson-McDermott EM, O'Neill LA. The Warburg effect then and now from cancer to inflammatory diseases. Bioessays. 2013;35(11):965-973.

15. Ullah MS, Davies AJ, Halestrap AP. The plasma membrane lactate transporter MCT4, but not MCT1, is up-regulated by hypoxia through a HIF-1alpha-dependent mechanism. J Biol Chem. 2006;281(14):90309037.

16. Lee MG, Pedersen PL. Glucose metabolism in cancer importance of transcription factor-DNA interactions within a short segment of the proximal region of the type II hexokinase promoter. $J$ Biol Chem. 2003;278(42):41047-41058.

17. Archer MC. Role of sp transcription factors in the regulation of cancer cell metabolism. Genes Cancer. 2011;2(7):712-719. 
18. Van Schaftingen E, Hers HG. Phosphofructokinase 2: the enzyme that forms fructose 2,6-bisphosphate from fructose 6-phosphate and ATP. Biochem Biophys Res Commun. 1981;101(3):1078-1084.

19. Minchenko OH, Ogura T, Opentanova IL, et al. Splice isoform of 6-phosphofructo-2-kinase/fructose-2,6-bisphosphatase-4: expression and hypoxic regulation. Mol Cell Biochem. 2005;280(1-2):227-234.

20. Minchenko $\mathrm{OH}$, Ochiai A, Opentanova IL, et al. Overexpression of 6-phosphofructo-2-kinase/fructose-2,6-bisphosphatase-4 in the human breast and colon malignant tumors. Biochimie. 2005;87(11):1005-1010.

21. Christofk HR, Vander Heiden MG, Harris MH, et al. The M2 splice isoform of pyruvate kinase is important for cancer metabolism and tumour growth. Nature. 2003;452(7184):230-233.

22. Warburg O. On respiratory impairment in cancer cells. Science. 1956;124(3215):269-270.

23. Samudio I, Fiegl M, Andreeff M. Mitochondrial uncoupling and the Warburg effect molecular basis for the reprogramming of cancer cell metabolism. Cancer Res. 2009;69(6):2163-2166.

24. Cairns RA, Harris IS, Mak TW. Regulation of cancer cell metabolism. Nat Rev Cancer. 2011;11(2):85-95.

25. Orimo A, Gupta PB, Sgroi DC, et al. Stromal fibroblasts present in invasive human breast carcinomas promote tumor growth and angiogenesis through elevated SDF-1/CXCL12 secretion. Cell. 2005;121(3):335-348

26. Untergasser G, Gander R, Lilg C, et al. Profiling molecular targets of TGF-beta1 in prostate fibroblast-to-myofibroblast transdifferentiation. Mech Ageing Dev. 2005;126(1):59-69.

27. Rønnov-Jessen L, Petersen OW, Koteliansky VE, et al. The origin of the myofibroblasts in breast cancer. recapitulation of tumor environment in culture unravels diversity and implicates converted fibroblasts and recruited smooth muscle cells. J Clin Invest. 1995;95(2):859-873.

28. Anderberg C, Pietras K. On the origin of cancer-associated fibroblasts. Cell Cycle. 2009;8(10):1461-1462.

29. Sumida T, Kitadai Y, Shinagawa K, et al. Anti-stromal therapy with imatinib inhibits growth and metastasis of gastric carcinoma in an orthotopic nude mouse model. Int J Cancer. 2011;128(9):2050-2062.

30. Direkze NC, Hodivala-Dilke K, Jeffery R, et al. Bone marrow contribution to tumor-associated myofibroblasts and fibroblasts. Cancer Res. 2004;64(23):8492-8495.

31. Petersen OW, Nielsen HL, Gudjonsson T, et al. Epithelial to mesenchymal transition in human breast cancer can provide a nonmalignant stroma. Am J Pathol. 2003;162(2):391-402.

32. Cannito S, Novo E, di Bonzo LV, et al. Epithelial-mesenchyma transition: from molecular mechanisms, redox regulation to implications in human health and disease. Antioxid Redox Signal. 2010;12(12):13831430

33. Zeisberg EM, Potenta S, Xie L, et al. Discovery of endothelial to mesenchymal transition as a source for carcinoma-associated fibroblasts. Cancer Res. 2007;67(21):10123-10128.

34. Pavlides S, Whitaker-Menezes D, Castello-Cros R, et al. The reverse Warburg effect: aerobic glycolysis in cancer associated fibroblasts and the tumor stroma. Cell Cycle. 2009;8(23):3984-4001.

35. Costa A, Scholer-Dahirel A, Mechta-Grigoriou F. The role of reactive oxygen species and metabolism on cancer cells and their microenvironment. Semin Cancer Biol. 2014;25:23-32.

36. Martinez-Outschoorn UE, Curry JM, Ko YH, et al. Oncogenes and inflammation rewire host energy metabolism in the tumor microenvironment: RAS and NFkB target stromal MCT4. Cell Cycle. 2013;12(16):2580-2597.
37. Martinez-Outschoorn UE, Sotgia F, Lisanti MP. Metabolic Asymmetry in Cancer: A "Balancing Act" that promotes tumor growth. Cancer Cell . 2014;26(1):5-7.

38. Stern R, Shuster S, Neudecker BA, et al. Lactate stimulates fibroblast expression of hyaluronan and CD44: the Warburg effect revisited. Exp Cell Res. 2002;276(1):24-31.

39. Whitaker-Menezes D, Martinez-Outschoorn UE, Lin Z, et al. Evidence for a stromal-epithelial "lactate shuttle" in human tumors: MCT4 is a marker of oxidative stress in cancer-associated fibroblasts. Cell Cycle. 2011;10(11):1772-1783.

40. Park JH, Kim TY, Jong HS, et al. Gastric epithelial reactive oxygen species prevent normoxic degradation of hypoxia-induciblefactorlalpha in gastric cancer cells. Clin Cancer Res. 2003;9(1): 433-440.

41. Ryu JH, Li SH, Park HS, et al. Hypoxia-inducible factoralpha subunit stabilization by NEDD8 conjugation is reactive oxygen speciesdependent. J Biol Chem. 2011;286:6963-6970.

42. Selak MA, Armour SM, MacKenzie ED, et al. Succinate links TCA cycle dysfunction to oncogenesis by inhibiting HIF-alpha prolyl hydroxylase. Cancer Cell. 2005;7(1):77-85.

43. Fiaschi T, Chiarugi P. Oxidative stress, tumor microenvironment, and metabolic reprogramming: a diabolic liaison. Int $J$ Cell Biol. 2012;2012:762825.

44. Black AR, Black JD, Azizkhan-Clifford J. Sp1 and Krüppel-like factor family of transcription factors in cell growth regulation and cancer. $J$ Cell Physiol . 2001;188(2):143-160.

45. Wierstra I. Sp1: emerging roles-beyond constitutive activation of TATA-less housekeeping genes. Biochem Biophys Res Commun. 2008;372(1):1-13

46. Rajakumar RA, Thamotharan S, Menon RK, et al. Sp1 and Sp3 regulate transcriptional activity of the facilitative glucose transporter isoform-3 gene in mammalian neuroblasts and trophoblasts. $J$ Biol Chem. 1998;273(42):27474-27483

47. Safe $\mathrm{S}$, Abdelrahim M. Sp transcription factor family and its role in cancer. Eur J Cancer. 2005;41(16):2438-2448.

48. Buzzai M, Bauer DE, Jones RG, et al. The glucose dependence of Akttransformed cells can be reversed by pharmacologic activation of fatty acid beta-oxidation. Oncogene. 2005;24(26):4165-4173.

49. Ramanathan A, Schreiber SL. Direct control of mitochondrial function by mTOR. Proc Natl Acad Sci U S A. 2009;106(52):22229-22232.

50. Ventura-Clapier R, Garnier A, Veksler V. Transcriptional control of mitochondrial biogenesis: the central role of PGC-1alpha. Cardiovasc Res. 2008;79(2):208-217.

51. Carracedo A, Ma L, Teruya-Feldstein J, et al. Inhibition of mTORC1 leads to MAPK pathway activation through a PI3K-dependent feedback loop in human cancer. J Clin Invest. 2008;118(9):3065-3074.

52. Menendez D, Shatz M, Resnick MA. Interactions between the tumor suppressor p53 and immune responses. Curr Opin Oncol. 2013;25(1):85-92

53. Basith S, Manavalan B, Yoo TH, et al. Roles of toll-like receptors in cancer: a double-edged sword for defense and offense. Arch Pharm Res. 2012;35(8):1297-1316.

54. Sato Y, Goto Y, Narita N, et al. Cancer cells expressing toll-like receptor and the tumor microenvironment. Cancer Microenviron. 2009;2(Suppl 1):205-214

55. Menendez D, Shatz M, Azzam K, et al. The Toll-like receptor gene family is integrated into human DNA damage and p53 networks. PLoS Genet. 2011;7(3):e1001360. 
56. Shatz M, Menendez D, Resnick MA. The human TLR innate immune gene family is differentially influenced by DNA stress and p53 status in cancer cells. Cancer Res. 2012;72(16):3948-3957.

57. Jacob J, Kelsoe G, Rajewsky K, et al. Intraclonal generation of antibody mutants in germinal centres. Nature. 1991;354(6352):389-392.

58. Hu JB, Zheng S, Deng YC. Expression of a novel immunoglobulin gene SNC73 in human cancer and non-cancerous tissues. World $J$ Gastroenterol. 2003;9(5):1054-1057.

59. Huang J, Sun X, Mao Y, et al. Expression of immunoglobulin gene with classical V-(D)-J rearrangement in mouse brain neurons. Int J Biochem Cell Biol. 2008;40(8):1604-1615.

60. Kimoto Y. Expression of heavy-chain constant region of immunoglobulin and T-cell receptor gene transcripts in human non-hematopoietic tumor cell lines. Genes Chromosomes Cancer. 1998;22(1):83-86.

61. Li J, Tan C, Xiang Q, et al. Proteomic detection of changes in protein synthesis induced by NGX6 transfected in human nasopharyngeal carcinoma cells. J Protein Chem. 2001;20(3):265-271.

62. Chen Z, Huang X, Ye J, et al. Immunoglobulin $\mathrm{G}$ is present in a wide variety of soft tissue tumors and correlates well with proliferation markers and tumor grades. Cancer. 2010;116(8):1953-1963.

63. Qiu Y, Korteweg C, Chen Z, et al. Immunoglobulin G expression and its colocalization with complement proteins in papillary thyroid cancer. Mod Pathol. 2012;25(1):36-45.

64. Qiu X, Zhu X, Zhang L, et al. Human epithelial cancers secrete immunoglobulin $\mathrm{g}$ with unidentified specificity to promote growth and survival of tumor cells. Cancer Res. 2003;63(19):6488-6495.

65. Zhang L, Hu S, Korteweg C, et al. Expression of immunoglobulin G in esophageal squamous cell carcinomas and its association with tumor grade and Ki67. Hum Pathol. 2012;43(3):423-434.

66. Zheng H, Li M, Liu H, et al. Immunoglobulin alpha heavy chain derived from human epithelial cancer cells promotes the access of $\mathrm{S}$ phase and growth of cancer cells. Cell Biol Int. 2007;31(1):82-87.

67. Hu D, Zheng H, Liu H, et al. Immunoglobulin expression and its biological significance in cancer cells. Cell Mol Immunol. 2008;5(5):319-324.

68. Wang J, Lin D, Peng H, et al. Cancer-derived immunoglobulin G promotes tumor cell growth and proliferation through inducing production of reactive oxygen species. Cell Death Dis. 2013;4:e945.

69. Chen Z, Gu J. Immunoglobulin G expression in carcinomas and cancer cell lines. FASEB J. 2007;21(11):2931-2938

70. Zheng H, Li M, Ren W, et al. Expression and secretion of immunoglobulin alpha heavy chain with diverse VDJ recombinations by human epithelial cancer cells. Mol Immunol. 2007; 44(9):2221-2227.

71. Babbage $\mathrm{G}$, Ottensmeier $\mathrm{CH}$, Blaydes $\mathrm{J}$, et al. Immunoglobulin heavy chain locus events and expression of activation-induced cytidine deaminase in epithelial breast cancer cell lines. Cancer Res. 2006;66(8):3996-4000.

72. Hsu LY, Lauring J, Liang HE, et al. A conserved transcriptional enhancer regulates RAG gene expression in developing B cells. Immunity. 2003;19(1):105-117.

73. Kishi H, Jin ZX, Wei XC, et al. Cooperative binding of $\mathrm{c}-\mathrm{Myb}$ and Pax-5 activates the RAG-2 promoter in immature B cells. Blood. 2002; 99(2):576-583.

74. Chen Z, Xiao Y, Zhang J, et al. Transcription factors E2A, FOXO1 and FOXP1 regulate recombination activating gene expression in cancer cells. PLoS One. 2011;6(5): e20475.

75. Lee BS, Dekker JD, Lee BK, et al. The BCL11A transcription factor directly activates RAG gene expression and V(D)J recombination. $\mathrm{Mol}$ Cell Biol . 2013;33(9):1768-1781
76. Jiang BY, Zhang XC, Su J, et al. BCL11A overexpression predicts survival and relapse in non-small cell lung cancer and is modulated by microRNA-30a and gene amplification. Mol Cancer. 2013;12:61.

77. Villarroel Dorrego M, Whawell SA, Speight PM, et al. Transfection and ligation of CD40 in human oral keratinocytes affect proliferation, adhesion and migration but not apoptosis in vitro. Clin Exp Dermatol. 2006;31(2):266-271.

78. Pinzon-Charry A, Maxwell T, McGuckin MA, et al. Spontaneous apoptosis of blood dendritic cells in patients with breast cancer. Breast Cancer Res. 2006;8(1):R5

79. von Leoprechting A, van der Bruggen P, Pahl HL, et al. Stimulation of CD40 on immunogenic human malignant melanomas augments their cytotoxic T lymphocyte-mediated lysis and induces apoptosis. Cancer Res. 1999;59(6):1287-1294.

80. Wingett DG, Vestal RE, Forcier K, et al.CD40 is functionally expressed on human breast carcinomas: variable inducibility by cytokines and enhancement of Fas-mediated apoptosis. Breast Cancer Res Treat. 1998;50(1):27-36

81. Hirano A, Longo DL, Taub DD, et al. Inhibition of human breast carcinoma growth by a soluble recombinant human CD40 ligand. Blood. 1999;93(9):2999-3007.

82. Posner MR, Cavacini LA, Upton MP, et al. Surface membrane-expressed CD40 is present on tumor cells from squamous cell cancer of the head and neck in vitro and in vivo and regulates cell growth in tumor cell lines. Clin Cancer Res. 1999;5(8):2261-2270.

83. Eliopoulos AG, Young LS. LMP1 structure and signal transduction Semin Cancer Biol. 2001;11(6):435-444.

84. Hu F, Zhang L, Zheng J, Zhao L, et al. Spontaneous production of immunoglobulin $\mathrm{M}$ in human epithelial cancer cells. PLoS One. 2012;7(12):e51423.

85. Tannahill GM, Curtis AM, Adamik J, et al. Succinate is an inflammatory signal that induces IL-1 $\beta$ through HIF-1 $\alpha$. Nature. 2013;496(7444):238242

86. Sukumar M, Liu J, Ji Y, et al. Inhibiting glycolytic metabolism enhances CD8+ T cell memory and antitumor function. $J$ Clin Invest 2013;123(10):4479-4488.

87. Swartz MA, Lund AW. Lymphatic and interstitial flow in the tumour microenvironment: linking mechanobiology with immunity. Nat Rev Cancer. 2012;12(3):210-219.

88. Sionov RV, Fridlender ZG, Granot Z. The multifaceted roles neutrophils play in the tumor microenvironment. Cancer Microenviron (Epub ahead of print). 2014.

89. Pearce EL, Pearce EJ. Metabolic pathways in immune cell activation and quiescence. Immunity. 2013;38(4):633-643.

90. Chouaib S, Janji B, Tittarelli A, et al. Tumor plasticity interferes with anti-tumor immunity. Crit Rev Immunol. 2014;34(2):91-102.

91. Santisteban M, Reiman JM, Asiedu MK, et al. Immune-induced epithelial to mesenchymal transition in vivo generates breast cancer stem cells. Cancer Res. 2009;69(7):2887-2895.

92. Billadeau DD, Burkhardt JK. Regulation of cytoskeletal dynamics at the immune synapse: new stars join the actin troupe. Traffic. 2006;7(11):1451-1460.

93. Abouzahr S, Bismuth G, Gaudin C, et al. Identification of target actin content and polymerization status as a mechanism of tumor resistance after cytolytic T lymphocyte pressure. Proc Natl Acad Sci U S A. 2006;103(5):1428-1433

94. Valencia T, Kim JY, Abu-Baker S, et al. Metabolic reprogramming of stromal fibroblasts through p62-mTORC1 signaling promotes inflammation and tumorigenesis. Cancer Cell. 2014;26(1):121-135. 
95. Godin-Ethier J, Hanafi LA, Duvignaud JB, et al. IDO expression by human B lymphocytes in response to T lymphocyte stimuli and TLR engagement is biologically inactive. Mol Immunol. 2011;49(1-2):253259.

96. Blache CA, Manuel ER, Kaltcheva TI, et al. Systemic delivery of Salmonella typhimurium transformed with IDO shRNA enhances intratumoral vector colonization and suppresses tumor growth. Cancer Res. 2012;72(24):6447-6456.

97. Metz R, Rust S, Duhadaway JB, et al. IDO inhibits a tryptophan sufficiency signal that stimulates mTOR: A novel IDO effector pathway targeted by D-1-methyl-tryptophan. Oncoimmunology. 2012;1(9):14601468.

98. Chang MY, Smith C, DuHadaway JB, et al. Cardiac and gastrointestinal liabilities caused by deficiency in the immune modulatory enzyme indoleamine 2,3-dioxygenase. Cancer Biol Ther. 2011;12(12):1050 1058 .

99. Silzle T, Kreutz M, Dobler MA, et al. Tumor-associated fibroblasts recruit blood monocytes into tumor tissue. Eur J Immunol. 2003;33(5):13111320 .

100. Balsamo M, Scordamaglia F, Pietra G, et al. Melanoma-associated fibroblasts modulate NK cell phenotype and antitumor cytotoxicity. Proc Natl Acad Sci U S A. 2009;106(49): 20847-20852.
101. Kraman M, Bambrough PJ, Arnold JN, et al. Suppression of antitumor immunity by stromal cells expressing fibroblast activation protein-alpha. Science. 2010;330(6005):827-830.

102. Hauzenberger D, Olivier P, Gundersen D, et al. Tenascin-C inhibits beta1 integrin-dependent $\mathrm{T}$ lymphocyte adhesion to fibronectin through the binding of its fnIII 1-5 repeats to fibronectin. Eur J Immunol. 1999;29(5):1435-1447.

103. Loike JD, Cao L, Budhu S, et al. Blockade of alpha 5 beta 1 integrins reverses the inhibitory effect of tenascin on chemotaxis of human monocytes and polymorphonuclear leukocytes through three-dimensional gels of extracellular matrix proteins. J Immunol. 2001;166(12):7534-7542.

104. Silzle T, Randolph GJ, Kreutz M, et al. The fibroblast: sentinel cell and local immune modulator in tumor tissue. Int J Cancer. 2004;108(2):173180 .

105. Kalluri R, Zeisberg M. Fibroblasts in cancer. Nat Rev Cancer. 2006;6(5):392-401.

106. Gerber PA, Hippe A, Buhren BA, et al. Chemokines in tumor-associated angiogenesis. Biol Chem. 2009;390(12):1213-1223.

107. Zhang Y, Li X, Zhang Q, et al. Berberine hydrochloride prevents postsurgery intestinal adhesion and inflammation in rats. $J$ Pharmacol Exp Ther. 2014;349(3):417-426. 